\title{
Hierarchical delineation and multivariate statistical discrimination of chemozones of the Cauvery Basin, south India: Implications on spatio-temporal scales of stratigraphic correlation
}

\author{
RAMKUMAR Mu. ${ }^{*}$, BERNER Z. ${ }^{2}$ and STÜBEN D. ${ }^{2}$ \\ ${ }^{1}$ Department of Geology, Periyar University, Salem - 636011, India; Present Address: Institute of Palaeoenvironment, \\ University of Erlangen-Nuernberg, D-91054 Erlangen, Germany \\ ${ }^{2}$ Institute of Mineralogy and Geochemistry, University of Karlsruhe, D-76128 Karlsruhe, Germany \\ (C) China University of Petroleum (Beijing) and Springer-Verlag Berlin Heidelberg 2010
}

\begin{abstract}
Success in locating oil pools in the Cauvery Basin, south India had been found to be based on the ability to delineate precisely the stratigraphic traps resulting from frequent sea level changes. However, recognition and delineation of them in terms of depositional units through conventional stratigraphic methods have been elusive owing to the limitations of such methods and lack of unified stratigraphic markers that could be traced at regional and basinal scale. This paper attempts to recognize depositional units in terms of chemozones, chronologic and lithostratigraphic units by assigning distinct geochemical signatures. Geochemical signatures were assigned through hierarchical delineation and discriminant function analysis. It is observed that individual depositional units could be recognized statistically with whole-rock geochemical composition. The strata under study show two second order chemozones comprising six major chemozones that in turn correspond to third order sea level cycles and minor chemozones at the scale of fourth order and/or further shorter sea level cycles. The geochemical signatures showed $100 \%$ distinctness between sample populations categorized according to chronostratigraphy and lithostratigraphy. The durations of these stratigraphic units range from 18 million years to less than a million years and indicate distinct geochemical compositional change at different time slices. By implication and also due to the close correspondence between sea level variations reported from this basin and global sea level cycles, it is suggested that recognition and correlation of individual depositional units with distal counterparts could be made accurately. Implication of these results is that stratigraphic units, at varying scales either temporally or spatially, could be assigned with unique geochemical signature, with which accurate prediction and correlation of similar units elsewhere is possible with measurable accuracy.
\end{abstract}

Key words: Chemostratigraphy, statistical discrimination, petroleum exploration, stratigraphic correlation

\section{Introduction}

As can be observed elsewhere except the Petroleum Exporting Countries, the widening gap between demand and production of petroleum in India has created a situation where unless discoveries of new oil fields are made, and higher recovery rates are achieved from the existing fields, the gap is poised to increase further. This increase would result in huge import bills and deplete foreign exchange reserves (Ramkumar, 1999). In turn, it would affect adversely economic and social development schemes, which no developing country could afford. Though the

*Corresponding author. email: muramkumar@yahoo.co.in Received May 18, 2009 east coast sedimentary basins of India are believed to be promising sources of hydrocarbon, the exploration efforts have so far failed to match the demand. Among these basins, recognition of paleo deltaic regimes is the key for targeting hydrocarbon sources in the Krishna-Godavari Basin, while high-resolution stratigraphic classification applicable for regional, onland and offshore equivalents of the rocks is the key for exploration and field development strategies in the Cauvery Basin (Govindan et al, 2000). The presence of many subtle stratigraphic traps created during the evolutionary history of the Cauvery Basin as a result of high-frequency sea level changes necessitates high-resolution stratigraphic understanding of the area for better exploration efforts. Occurrences of patchy and locally concentrated faunal population, barren interbeds and extensive exotic blocks 
in this basin have thwarted stratigraphic correlation using conventional methods (Ramkumar et al, 2004).

During the last two decades, chemostratigraphy has emerged to be a potential tool for correlation of widely separated strata where other conventional stratigraphic methods fail or have limitations (Ramkumar and Sathish, 2006). Many publications are available concerning chemostratigraphic application and its potential as a vital tool for stratigraphic correlation, fixation of geological boundaries and petroleum exploration. Those studies attempted to solve selective boundary questions and focused only on documenting shifts or trends of geochemical and or isotopic composition across a chronological boundary or strata of limited chronological span with a few selective elemental or isotopic data.

The stratigraphic record is the outcome of an exogenic system consisting of geologic setting, changes in sea level, changes in geochemical reactions between the sea and earth, climate and processes of sediment formation. Hence, the ensuing sedimentary records show differences in bulk chemical composition as the changes produce different combinations of minerals, primary differences in the composition of their constituent minerals or in the proportions of accessory phases such as heavy minerals and clays, many of which have distinctive major and trace element contents (Das, 1997). Judging from these differences and based on the distinctness of chemical composition and its causative factors (local, regional or global), the chemozones should be recognized and correlated at an appropriate level (Ramkumar, 1999; Ramkumar and Sathish, 2006). Thus, applicability of chemostratigraphic technique as a potential tool for stratigraphic correlation can be tested only when its perceived capabilities are examined critically through analysis of wholerock geochemical trends of strata representing considerable time span. In addition, the ability of whole-rock chemical analysis to distinguish depositional units produced under varying conditions of sedimentation has to be demonstrated. Thus, this paper attempts to characterize the depositional units of the Barremian-Danian strata of the Cauvery Basin through discrimination of their whole-rock geochemical composition.

\section{Geologic setting}

The Cauvery Basin (Fig. 1) was created during Late Jurassic-Early Cretaceous rifting associated with fragmentation of the Gondwana super continent. Table 1 presents the lithostratigraphy (Ramkumar et al, 2004) and gross lithological information of this basin. Geological details of the study area are presented in Fig. 1. Sedimentation in this basin commenced in shallow water environments. Most of the deposits of this basin represent deposition in a shallow epicontinental sea and are punctuated with slope and basinal deposits. While the older deposits are characterized by dynamic environmental changes and texturally immature siliciclastics, younger deposits are characterized by comparatively stable depocenters, carbonate deposition and textural inversion and maturity. At each major unconformity, granitic and granitic gneiss basement rocks and older sedimentary rocks were eroded and transported to newer depocenters to deposit lithoclastic conglomerates. Lithofacies associations and fossil data indicate the basis was periodically sediment-starved. Whenever the siliciclastic influx was restricted and/or ceased, carbonate sedimentation was initiated immediately in the basin. Occurrence of contact between very old deposits and much younger deposits in the lower half of the rock record and absence of such features in younger strata indicate reduced significance of tectonic movements in the latter part of the evolutionary history of the basin. After initial block faulting and inception of sedimentation during the Late Jurassic-Early Cretaceous, the intensity of tectonic control over sedimentation diminished, although the basin continued evolving till the end of the Tertiary (Prabhakar and Zutshi, 1993). The sedimentation history of the basin was influenced by eustatic sea level changes of 3rd order cycles, within which many higher order cycles are embedded (Raju et al, 1993; Ramkumar et al, 2004). Record of all the seven global sea level peaks in this basin affirms close links between the depositional history of this basin and global cycles.

\section{Materials and methods}

Systematic field mapping at a scale of 1:50,000 was conducted in the erstwhile Tiruchirapalli district where a complete and extensive exposure of the Barremian-Danian strata of the Cauvery Basin is accessible for close examination and sampling. Ten traverses were made (Fig. 1) and a total of 308 locations were logged and sampled. A composite stratigraphic profile representing Barremian-Danian rock record was constructed. From this 157 rock samples were selected for whole-rock trace element analysis. Among these 157 samples, 70 samples were also analyzed for major elements. Trace and major element analyses were performed by X-ray fluorescence analysis (XRF) (as discussed in Stüben et al, 2002; Ramkumar et al, 2006). Analyses of 157 samples for bulk mineralogical and 70 samples for clay mineralogical compositions were also performed. This paper is based only on the geochemical data while mineralogical and petrographic observations were supplementary to the inferences from geochemistry.

As the stratigraphic record is a product of the geochemical system consisting of geologic setting, climate and processes of sediment production in a geomorphic setting, variations in depositional conditions operated under different scales through time might produce distinct geochemical signatures (chemostratigaphic indices) for individual depositional units (chemozones). These chemozones could be recognized and used for correlation with comparable stratigraphic section of the basin. However, geochemical profiles of the Cauvery Basin have shown a complex and subtle array of signals as a result of multitudes of depositional episodes dominated by a single geological factor namely, sea level fluctuations (Ramkumar et al, 2010, in press). These authors have examined the bulk geochemical data in terms of absolute concentrations and geochemical profiles and found them too complex and subtle to be recognized as individual chemozones. If insufficient sampling coverage is assumed to be the cause of this state-of-affairs, representation of $\sim 1,300 \mathrm{~m}$ thick strata through 157 samples translates into more than 


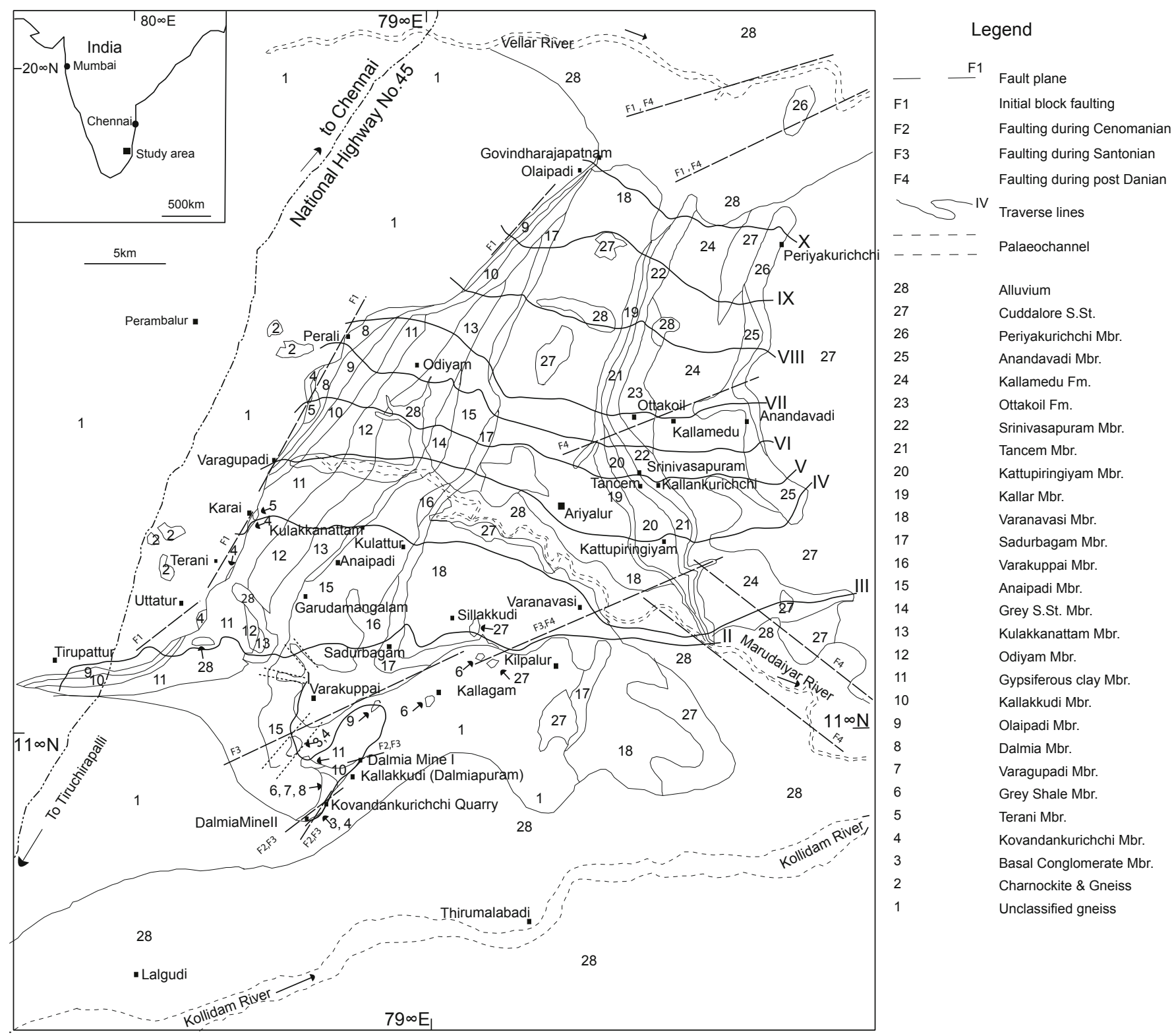

Fig. 1 Location of the Cauvery Basin, geology of the study area and traverses along which samples were collected

one sample per every ten meters of strata. This is compatible with the sampling frequency followed for exploratory drilling practiced in this part of the country. As 157 samples represent 56.5 million years of sedimentary record, every millionyear record is covered by 2.78 samples. In this account also, the sampling interval is adequate with reference to chronological units (ranging between 1 and 15.5 million years) or lithostratigraphic formations (ranging between 2 and 18 million years) or lithostratigraphic members (ranging between less than a million year and few million years). It is presumed that, although the depositional units are geochemically different (as indicated by analysis of variance (ANOVA), Ramkumar et al, 2010, in press), manual distinction of these units was found to be difficult due to the complex and subtle geochemical signals (Ramkumar et al, 2006). As the depositional history and elemental composition of the studied rocks were controlled by global sea level and climatic fluctuations, the facies succession of this basin could be considered as depositional sequences (sensu Vail et al, 1977) and hence, it is felt that the depositional units have to be tested for their distinct chemical signatures. If these units are found to be geochemically distinct, they can be treated as high-resolution chemozones. Our previous publications have discussed the facies characteristics (Ramkumar et al, 2004), stratigraphic variations of geochemical data (Ramkumar et al, 2010 , in press) and relationships between geochemistry and facies characteristics (Ramkumar et al, 2006). In this paper, we attempt to characterize the strata using a statistical tool namely Discriminant Function Analysis (DFA).

The purpose of statistical characterization is to determine whether the geological populations (samples of depositional units categorized in terms of chronology, lithostratigraphy or chemostratigraphy) have distinct geochemical compositions. It is to deduce the inter-population variability of different parameters (concentrations of geochemical elements of various depositional units) with a view to differentiate 
Table 1 Lithostratigraphy of the Cauvery Basin (after Ramkumar et al, 2004) and the codes utilized for discrimination of depositional units

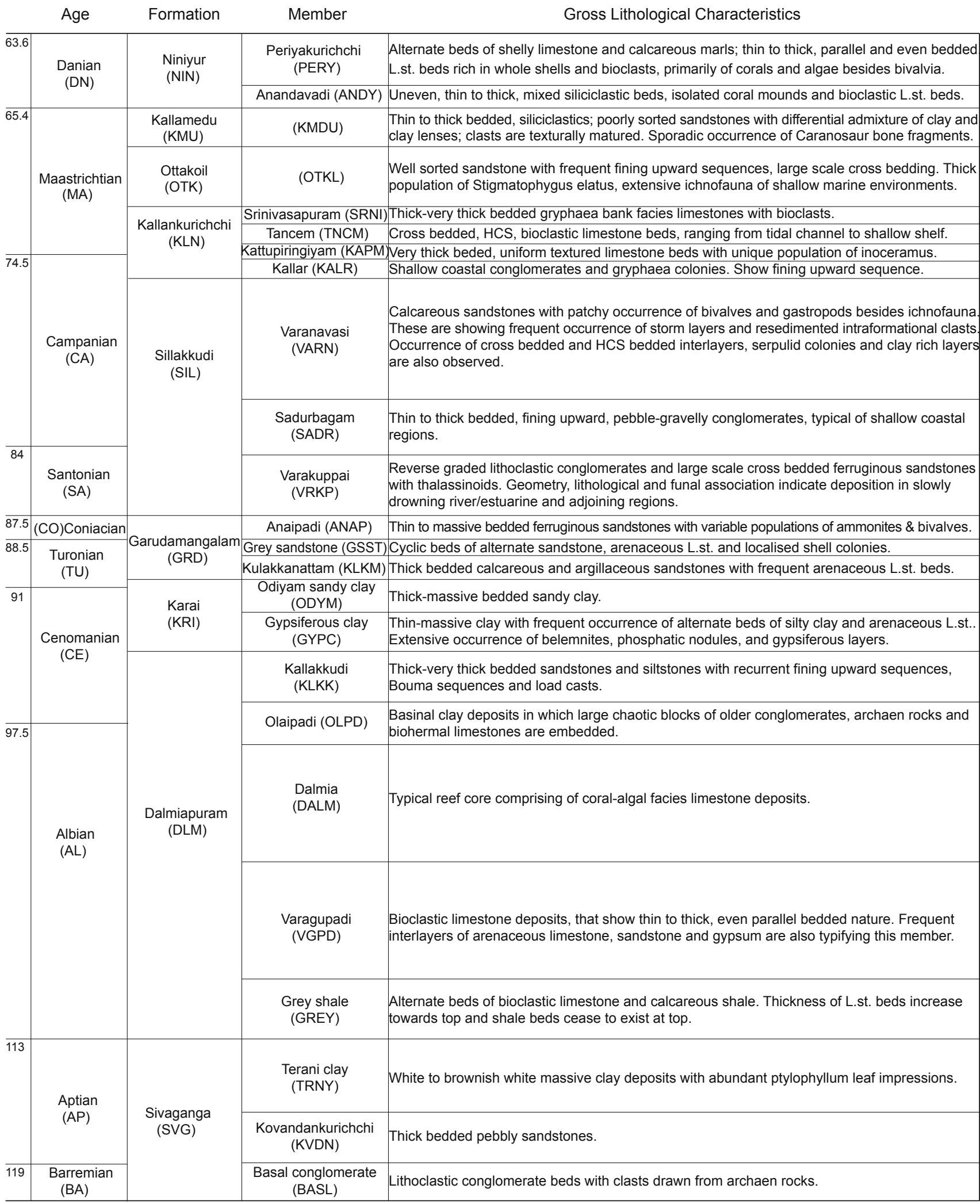

statistically (vis-à-vis geochemically) individual depositional units from each other. To group samples for identifying distinct populations from a widely ranging dataset, Q-mode cluster analysis is commonly utilized. Loizeau and Stanley (1993) are of the opinion that the grouping established by cluster analysis has to be tested by DFA. Effective handling of large datasets and large numbers of populations to be tested for their distinctness could not be accomplished by cluster analysis alone (Ramkumar, 2001). Since cluster analysis lacks prediction capability, recognition of the existing populations 
through geochemical composition and identification of related populations from unknown samples was undertaken by DFA instead.

DFA is the classification procedure of choice when known populations of objects must be separated on the basis of a set of constituent variables. Roser and Korsch (1988) used DFA successfully to test provenance signatures of rocks based on whole-rock geochemistry. Applying DFA, Ferrell et al (1998) differentiated the sedimentary environments of the Krishna delta, India, based on whole-rock mineralogical compositions. Pearce et al (1999) have tested distinctness of chemozones through DFA. DFA was successfully applied by Ramkumar and Guha (2000) to discriminate standard microfacies and lithostratigraphic members based on petrographic point count data of Tertiary carbonates of Kutchch region of India. Differences in textural and geochemical composition of Godavari deltaic sediments were utilized to distinguish and predict the depositional environment of unknown samples with the help of DFA to the tune of $76 \%-100 \%$ accuracy (Ramkumar, 2001). A scheme of petrographic type recognition and prediction involving DFA based on petrographic and geochemical data from Maastrichtian carbonates of the Cauvery Basin was evolved by Ramkumar et al (2002). These studies have upheld the potential of DFA to recognize and distinguish geological populations.

DFA derives a set of linear functions of multiple variables designed to achieve best separation between predefined groups (Roser and Korsch, 1988). It is valuable for analysis of complex datasets for which interactions among variables are too complex (Ferrell et al, 1998) such as cluster analysis (Ramkumar, 2001). Thus, the fundamental questions asked while performing statistical characterization are (modified from Ferrell et al, 1998): Do the chronological and lithostratigraphic units and chemozones have distinct geochemical signatures? If yes, could they be recognized through statistical procedures? If proved positive, which are the elements that show significant difference between various depositional units? How distinct these geochemical signatures are? Could they be utilized for recognition of similar units? Answering these questions would help evolve a comprehensive scheme to distinguish depositional units through geochemical signatures.

As DFA requires the samples under study to be categorized prior to the analysis, which (chronologic and lithostratigraphic units and chemozones) in turn would be tested for uniqueness, each sample was assigned with an appropriate code in the computer data file. Table 1 lists the respective codes (given in parentheses) for different groups that were discriminated. A total of 47 variables drawn from whole-rock geochemical analyses, calculation of ratios of a few selected elements and relative sea level estimates for each sample (based on depositional environmental interpretation and faunal data) were added with the geochemical database and utilized for discrimination. Measurement units of these geochemical and other variables are different, in which case, many authors (Nayak et al, 1997; Ramkumar, 2001; Ramkumar and Guha, 2000) suggested an appropriate transformation prior to any meaningful statistical analyses. Thus, the entire dataset was standardized with the transform function $z=(x-m) / s$ (where $x$ is the value of the random variable; $m$ is mean of the random variable; $s$ is standard deviation; $z$ is standardized variable). Contrary to the widely scattered raw data, the standardized values have a narrow range and mean value of zero and standard deviation of 1 . For all statistical analyses of this study, only the standardized dataset is utilized. Statistical analyses were performed with STATISTICA software produced by Stat Soft Inc., U.S.A.

\section{Results and interpretations}

\subsection{Discriminant capability of individual elements}

Many classic and recent chemostratigraphic studies utilized only selected elemental concentrations for defining chemostratigraphic indices under the notion that only few elements could serve the purpose. Elucidation of discrimination capacity of individual elements would help decide whether only a few elemental concentrations, or the whole set of chemical data are necessary for chemostratigraphic studies. Hence, DFA was performed with each element separately to document presence/absence of a distinct geochemical signature for individual depositional units (chronologic and lithostratigraphic units).

Tables 2(a), 2(b) and 2(c) present the discrimination percentages of geochronological units, lithostratigraphic formations and members respectively, based on individual geochemical parameters. These tables show that nearly all the elements serve as discriminants, albeit with poor discrimination power. For example, the Table 2(a) shows that only $22.1 \%$ of discrimination is possible for chronological units based on a single parameter. Among the chronological age units, only Maastrichtian shows a higher average discrimination of $84.6 \%$. Among individual elements, only $\mathrm{Y}$ and Mn show more than $25 \%$ discrimination capacity. While analyzing the factors that influenced variance of the data of samples used in this present study, Ramkumar et al (2006) recorded that these two elements were brought to the depocenter through detrital influx and their variance ranks next only to the influence of sea level. The discriminating capability of these two elements as showed by relatively higher percentages than all other elements suggests preservation of depositional signatures in these rocks and distinct change in quantum of influx of these elements with the passage of time. Ba shows least discrimination, may be because of its influx as Ba-orthoclase (as indicated by X-ray diffractograms, Ramkumar et al, 2006) into the depocentre immediately after every major unconformity and repeated deposition of lithoclastic conglomerates in which Ba-orthoclase forms significant proportion. Its ubiquitous presence in many of the depositional units might have resulted in generation of geochemical indifference between the depositional units.

It appears from the Table 2(b) that the average discrimination percentage of formations is slightly better than the geological age units. Although the discrimination percentage is only marginally higher $(27.3 \%)$, such an increase could be interpreted as indicative of a more significant shift of geochemical compositions across 
Table 2(a) Discrimination percentages of geochronological units

\begin{tabular}{|c|c|c|c|c|c|c|c|c|c|c|c|c|c|c|c|c|c|c|c|c|c|c|c|c|c|c|c|c|c|c|c|c|c|c|c|c|c|c|c|c|}
\hline & & $\mathrm{Si}$ & & & $\mathrm{Mg}$ & P & K & & Ti & n & LOI & I $\mathrm{s}$ & & $\mathrm{CO}_{2}$ & $\mathrm{C}_{\text {inovg }} \mathrm{C}$ & $\mathrm{C}_{\mathrm{cog}} \mathrm{N}$ & $\mathrm{Zn} \quad \mathrm{A}$ & As & $\mathrm{Br}$ & $\mathrm{Rb}$ & $\mathrm{Sr}$ & & & $\mathrm{Nb} \quad \mathrm{N}$ & Mo A & $\mathrm{Sn} \quad \mathrm{Sb}$ & & & $\mathrm{La}$ & & $\mathrm{Pb}$ & & $\begin{array}{l}\mathrm{Mg} / \mathrm{I} \\
\mathrm{Ca}\end{array}$ & In/ 1 & $\begin{array}{l}e / 1 \\
a\end{array}$ & & $\begin{array}{ll}\mathrm{Na} / & \mathrm{Z} \\
\mathrm{Ca}\end{array}$ & & & \\
\hline & & & & & & 0.0 & 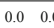 & & & & & & & 0.0 & 00 & & $\begin{array}{ll}0.0 & 0\end{array}$ & 00 & 0.0 & 0.0 & 0.0 & & & & & & & & & & & & & & & & & & & \\
\hline & & & 78.67 & 67 & 78.692 & 92.975 & 75.082. & & & 0.078. & & & & 78.6 & $\begin{array}{ll}78.65 \\
5\end{array}$ & 50.07 & & 75.01 & 100.078 & 78.68 & 85.792 & 92.99 & 92. & & & & & 4 & & & & 49 & 96.4 & & & & & & & \\
\hline $\mathrm{CA}$ & & & 00 & 00 & 10 & $0.0 \quad 18$ & $\begin{array}{lll}18.8 & 0.6\end{array}$ & & 100 & 0 & $\begin{array}{ll}0 & 0.0\end{array}$ & 0.0 & 0.0 & 0.0 & 0.0 & & $\begin{array}{lll}0 & 0.0 & 12\end{array}$ & 12.5 & $\begin{array}{ll}0.0 & 18\end{array}$ & 18.80 & 0.0 & 00 & & $\begin{array}{ll}0 & 0\end{array}$ & 000 & $0.0 \quad 0.0$ & $\begin{array}{ll}0 & 0.0\end{array}$ & 0.0 & 0.01 & 18.8 & $\begin{array}{ll}0.0 & 0.0\end{array}$ & 0.0 & 0.0 & 0.00 & 0.0 & $\begin{array}{ll}0 & 0\end{array}$ & $\begin{array}{cc}0.0 & 0 .\end{array}$ & & $\begin{array}{ll}.0 & 0.0\end{array}$ & \\
\hline$S A$ & & & & & & & & & & & & 014. & & & & & & & & & & & & & & & & & & & & & & & & & & & & \\
\hline 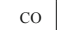 & & & & & & & & & & & $\begin{array}{ll}7 & 0.0\end{array}$ & 0.0 & & & & & & & & & & & & & & & & & & & & & & 10 & 10 & & & & & \\
\hline & & & & 62.5 & & & & & & & & & & & & & & & & & & & & & & & & & & & & & & & & & & & & \\
\hline $\mathrm{CE}$ & & 36.44 & 45.5 & 0.0 & & & 41.758. & & 0.8 & & .727 .3 & 30.0 & 35 & 58.3 & 58.3 & & & 0.0 & $4.2 \quad 45$ & $45.8 \quad 2$ & 25.00 & & & & & & 320.8 & 50.0 & 0.0 & 0.0 & & & & 0.0 & $\begin{array}{ll}0 & 0\end{array}$ & & 0.0 & 4.2 & 0 & 21 \\
\hline & & & & & & & & & & 36. & & & & 20.0 & 20 & & & & & & & & & & & & & & 6.04 & & & & & & & & & & & \\
\hline & & & & & & & & & & $\begin{array}{ll}30.0 \\
\end{array}$ & .00 & 0. & & & & & & & & & & & & & & $\begin{array}{ll}0.0 & 0.0\end{array}$ & $\begin{array}{ll}0 & 0.0\end{array}$ & 0 & 0.0 & $\begin{array}{ll}0.0 & 1\end{array}$ & & 0 & 2.9 & 0.0 & .0 & & $\begin{array}{lll}0.0 & 28\end{array}$ & 28.61 & & \\
\hline & & & & & & & & & & & & & & & & & & & & & & & & & & & & & & & & & & & & & & & & \\
\hline & & & & & & & 21. & & & & & 118 & 26.12 & 26.1 & 3 & & & & & 24.22 & 432 & 32 & & & 22 & 2.31 & & & & & & & & & & & & & & \\
\hline
\end{tabular}

Table 2(b) Discrimination percentages of lithostratigraphic formations

\begin{tabular}{|c|c|c|c|c|c|c|c|c|c|c|c|c|c|c|c|c|c|c|c|c|c|c|c|c|c|c|c|c|c|c|c|c|c|c|c|c|c|c|c|c|c|c|c|c|}
\hline & & $\mathrm{Si}$ & Al & $\mathrm{Na}$ & $\mathrm{Mg}$ & $\mathrm{P}$ & $\mathrm{K}$ & & T & 1 & $\mathrm{Fe}$ & LOI & & & & $C_{\text {inoss }}$ & $C_{\text {og }}+1$ & & & As & $\mathrm{Br}$ & $\mathrm{Rb}$ & $\mathrm{Sr}$ & Y & $\mathrm{Zr}$ & $\mathrm{Nb} \quad \mathrm{N}$ & Mo $\mathrm{Ag} \mathrm{Cd} \mathrm{Sn}$ & $\mathrm{Sn}$ Sb & I & $\mathrm{Ba}$ & a $\mathrm{La}$ & $\mathrm{Ce}$ & $\mathrm{Pb}$ & & & & $\begin{array}{l}\mathrm{Mn} / \\
\mathrm{Ca}\end{array}$ & & & $\begin{array}{l}\mathrm{Na} / \\
\mathrm{Ca}\end{array}$ & & & & \\
\hline & & & & 0.0 & & & 0.0 & 0.0 & 0.0 & & & 0.0 & & & 0.0 & 0.0 & 0.00 & $\begin{array}{ll}0.0 & 0.0\end{array}$ & 05.6 & & & & & & & & $\begin{array}{llll}0.0 & 0.0 & 0.0 & 0.6\end{array}$ & & & & & & & & & .0 & 0.0 & 0.0 & .0 & & .0 & 0.0 & & \\
\hline & & & & & & & & & & & & & & & & & & & & & & & & & & & & & & & .0 & & & & & & 0.0 & 10 & & & & 0.0 & 0 & \\
\hline & & & & & & & & & & & & & & & & & & & & & & & & & & & & & & & & & & & & & & & & & & & & \\
\hline & & & 63.6 & 0. & & & & 66.7 & & 29.2 & 20.87 & $72.7 \mathrm{c}-\mathrm{r}=\mathrm{s}$ & 0.0 & & 70.8 & 70.8 & & & & & & & & 33.3 & & & & & 0. & & .0 & & & & & & 0.0 & & & & 0.0 & & & \\
\hline & & & & & & & & & & & & & & & & & & & & & & & & & & & & & & & & & & & & & & & & & & & & \\
\hline & & 20. & 40.0 & 70.0 & 0.0 & & 22.2 & 7.4 & 91 & 18.5 & 2 & $20.0 \mathrm{c}$ & 0.0 & & 11.1 & 11.1 & & & & 0.0 & 9 & 22 & 0 & & & & & & & & 13.7 & 0.0 & & & & & 11.13 & 3.7 & & & 0.0 & $29.6 \mathrm{c}$ & & \\
\hline & & & 00 & & & & & & & & & & & & & & & & & & & & & & & & & & & & & & & & & & & & & & & & & \\
\hline & & & & & & & & & & & & & & & & & & & & & & & & & & & & & & & & & & & & & & & & & & & & \\
\hline & & & & & & & & & & & & & & & & & & & & & & & & & & & & & & & & & & & & & & & & & & & & \\
\hline
\end{tabular}

Table 2(c) Discrimination percentages of lithostratigraphic members

\begin{tabular}{|c|c|c|c|c|c|c|c|c|c|c|c|c|c|c|c|c|c|c|c|c|c|c|c|c|c|c|c|c|c|c|c|c|c|c|c|c|c|c|c|c|c|c|c|}
\hline oups 1 & & & Al & $\mathrm{Na}$ & $\mathrm{Mg}$ & $\mathrm{P}$ & $\mathrm{K}$ & $\mathrm{Ca}$ & $\mathrm{Ti}$ & $\mathrm{Mn}$ & $\mathrm{Fe} \mathrm{I}$ & LOI & s & $C_{\text {bot }}$ & & $C_{\text {ind }}$ & $\mathrm{C}_{\mathrm{vog} g}$ & $\mathrm{NiCu}$ & & As & $\mathrm{Br}$ & $\mathrm{Rb}$ & $\mathrm{Sr}$ & $\mathrm{Y}$ & $\mathrm{Zr}$ & $\mathrm{Nb}$ & Mo $\mathrm{Ag} \mathrm{Cd} \mathrm{Sn} \mathrm{St}$ & Sb $\quad \mathrm{I}$ & $\mathrm{Ba}$ & $\mathrm{La}$ & $\mathrm{Ce}$ & $\mathrm{Pb}$ & & & $\begin{array}{l}\mathrm{Mg} / \\
\mathrm{Ca}\end{array}$ & $\begin{array}{l}\mathrm{Mn} / \\
\mathrm{Ca}\end{array}$ & $\begin{array}{l}\mathrm{Fe} / \\
\mathrm{Ca} \\
\end{array}$ & $\begin{array}{ll}\mathrm{Ba} / & \mathrm{N} \\
\mathrm{Ca} & \mathrm{C} \\
\end{array}$ & $\begin{array}{l}\mathrm{Na} / \\
\mathrm{Ca} \\
\mathrm{Ca}\end{array}$ & $\begin{array}{l}\mathrm{Zn} /^{2} \\
\mathrm{Ca}\end{array}$ & & & \\
\hline RY & & & 0.0 & 50 & 25.0 & 100.0 & 7.7 & 23.1 & 0.01 & 100.0 & 92.3 & 0.0 & $0.0 \quad 30$ & 30.83 & 30.8 & 30.8 & 38.5 & 7.769 .2 & 292.31 & 15.4 & 0.05 & 53.8 & 38.5 & 76.9 & 7.7 & 53.8 & 7.730 .80 .038 .546 & 46.20 .0 & $\begin{array}{ll}0 & 0.0\end{array}$ & 61.5 & 569.23 & & 6 & & 0.0 & 84.6 & 0.00 & $\begin{array}{lll}0.0 & 0\end{array}$ & 0.00 & 0.00 & 106 & 231. & 31.8 \\
\hline & & & 0.0 & & 0.0 & & 0.0 & 0.0 & & 0.0 & 0.06 & & 0.0 & 0.0 & 0.0 & 0.0 & & c & 0.0 & 0.0 & .0 & 0.0 & 0.0 & 0.0 & & 0.0 & 0.0 & $\begin{array}{ll}0.0 & 0.0 \\
-\end{array}$ & 0.0 & 0.0 & 0.0 & 0.0 & 0.0 & .0 & 0.0 & 0.0 & 0.00 & 0.00 & 0.0 & 0.0 & 0.00 & 0.0 & 2.8 \\
\hline & 0.0 & 0.0 & 0.0 & 0.0 & 0.0 & & 0.0 & 0.0 & & 20.0 & 0.0 & 0.0 & $\begin{array}{ll}0.0 & 0 \\
0\end{array}$ & 0.0 & 0.0 & 0.0 & 0.0 & 0.00 .0 & 0.0 & 0.0 & 0.0 & 0.0 & 0.0 & 0.0 & 0.0 & 0.0 & $\begin{array}{cccccc}0.0 & 0.0 & 0.0 & 0.0 & 0 .\end{array}$ & $0.0 \quad 0.0$ & 0.0 & 0.0 & 0.0 & 0.0 & 0.0 & 0.0 & 0.0 & 40.0 & 0.00 & 0.00 & 0.0 & $0.0 \quad 0$ & 0.0 & $0.0 \quad 1$. & 1.3 \\
\hline & & & & & & & & & & & 0 & 0.0 & 0.0 & & 0.0 & 0.0 & 0 & 0.00 & 0.0 & 0.0 & 0.0 & 0.0 & 0.0 & 0.0 & 33.3 & 0.0 & $\begin{array}{lllll}33.3 & 0.0 & 0.0 & 0.0 & 0 .\end{array}$ & $0.0 \quad 0.0$ & $\begin{array}{ll}0 & 16.7\end{array}$ & 716.7 & 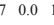 & 16.7 & 0.0 & .0 & 0.0 & 0.0 & 0.00 & 0.00 & 0.0 & 0.00 & 0.0 & 0.0 & 2. \\
\hline SRNI & $\mid 0.0$ & 0.0 & 0 & 0.0 & 0.0 & & .0 & & & & 0.0 & 0.0 & $0.0 \quad 0$ & 0.0 & 0.0 & 0.0 & 0.0 & $\begin{array}{ll}.00 & 0.0\end{array}$ & 0.0 & 0.0 & 0.0 & 0.0 & 0.0 & 0.0 & 0.0 & 0.0 & $\begin{array}{llllll}0.0 & 0.0 & 0.0 & 0.0 & 0 .\end{array}$ & $0.0 \quad 0.0$ & 0.0 & 0.0 & 0.0 & 0.0 & 0.0 & 0.0 & 0.0 & 0.0 & 0.00 & 0.00 & 0.0 & $\begin{array}{l}0.0 \\
0\end{array}$ & 0.0 & 0.0 & 0.0 \\
\hline & & 0.0 & 0.0 & 0.0 & 0.0 & & 0.0 & 0.0 & .0 & 0.0 & 0.0 & 0.0 & $\begin{array}{ll}0.0 & 0 \\
0\end{array}$ & 0.0 & 0.0 & 0.0 & 0.0 & $\begin{array}{lll}0.0 & 0.0\end{array}$ & 0.0 & 0.0 & 0.0 & 0.0 & 0.0 & 0.0 & 0.0 & 0.0 & $\begin{array}{cccccc}0.0 & 0.0 & 0.0 & 0.0 & 0 .\end{array}$ & $0.0 \quad 0.0$ & 0.0 & 0.0 & 0.0 & 0.0 & 0.0 & 0.0 & 0.0 & 0.0 & 0.00 & 0.00 & 0.0 & 0.00 & 0.0 & $0.0 \quad 0$. & 0.0 \\
\hline IPM & 75.01 & 100.07 & 75.0 & 75.01 & 100.0 & 25.0 & 50.0 & 37.5 & 75.0 & 0.0 & 12.55 & 50.0 & $0.0 \quad 50$ & 50.05 & 50.0 & 50.0 & 75.0 & 0.050 .0 & 037.57 & 75.0 & 0.05 & 50.0 & 62.5 & 37.5 & 87.5 & 62.5 & 37.525 .00 .025 .025 & $25.0 \quad 0.0$ & $\begin{array}{ll}0 & 0.0 \\
-\end{array}$ & 50.0 & 012.5 & 0.0 & 0.0 & 0.0 & 0.0 & 0.0 & 0.00 & 0.00 & 0.0 & 0.0 & 0.00 & $0.0 \quad 32$ & 32.7 \\
\hline ALR & 0.0 & 0.0 & 0.0 & 0.0 & 0.0 & 0.0 & 0.0 & 0.0 & 0.0 & 0.0 & 0.0 & 0.0 & $\begin{array}{ll}0.0 & 0 \\
-1\end{array}$ & 0.0 & 0.0 & 0.0 & 0.0 & 0.0 & 0.0 & 0.0 & 0.0 & 0.0 & 0.0 & 0.0 & 0.0 & 0.0 & $\begin{array}{cccccc}0.0 & 0.0 & 0.0 & 0.0 & 0 .\end{array}$ & $0.0 \quad 0.0$ & 0.0 & 0.0 & 0.0 & 0.0 & 0.0 & 0.0 & 0.0 & 0.0 & 0.00 & 0.00 & 0.0 & 0.00 & 0.00 & $0.0 \quad 0$. & 0.0 \\
\hline & 0.0 & 33.3 & 0.0 & & & & 44.4 & 0.0 & & .0 & 0.0 & 0.0 & $\begin{array}{ll}.0 & 0 \\
0 & \end{array}$ & 0.0 & 0.0 & 0.0 & 04 & 44 & 0.0 & 0.0 & 0.05 & 55.6 & 0.0 & 11.1 & 0.0 & 0.0 & $\begin{array}{lllll}11.1 & 0.0 & 0.0 & 0.0 & 0 .\end{array}$ & $0.0 \quad 0.0$ & 0.0 & .0 & & 0 & & 0 & 0.0 & 0.0 & & 22.211 & & 0.00 & 0.00 & 0.0 & 6.4 \\
\hline ADR & 0.0 & 0.0 & & & & & 0.0 & 0 & 5.0 & 0.0 & 0.0 & 0.02 & 25.00 & 0.0 & 0.0 & 0.0 & 0 & $\begin{array}{lll}0.0 & 0.0\end{array}$ & 0.0 & 0.0 & 0.0 & 0.0 & 0.0 & 0.0 & 0.0 & 0.0 & $\begin{array}{lllll}0.0 & 0.0 & 0.0 & 0.0 & 0 .\end{array}$ & $0.0 \quad 0.0$ & $\begin{array}{ll}0 & 0.0\end{array}$ & 0.0 & 0 & 0. & 0.0 & 25.0 & 0.0 & 0.02 & 25.00 & 0.00 & 0.0 & 0.0 & 0.0 & 0.02 & \\
\hline /RKP & 0.0 & 0.0 & 0 & & & & 0.0 & 0.0 & & & 0 & 0.0 & .0 & 10 & 0.0 & 0.0 & 0.0 & $\begin{array}{lll}0.0 & 0.0\end{array}$ & 0.0 & 0.0 & 0.0 & 0.0 & 0.0 & 0.0 & 0.0 & 0.0 & $\begin{array}{lll}0.0 & 0.0\end{array}$ & $0.0 \quad 0.0$ & 0.0 & 0.0 & 0.0 & 0.0 & 0.0 & .0 & 0.0 & 0.0 & 0.03 & & 0.0 & 0.0 & 0.0 & $\begin{array}{ll}0.0 & 0\end{array}$ & 0.7 \\
\hline $\mathrm{Al}$ & 0.0 & 0.02 & 25.0 & 0. & .0 & .0 & 53.3 & 0.0 & & 320.0 & 0.0 & 0.0 & .0 & & 0 & 0.0 & & 13.3 & 0.0 & 0.0 & 13.33 & 33.3 & 40.0 & 53.3 & 0.0 & 46.7 & 0.0 & 40.06 .7 & 3.3 & 3 & & 6.7 & & & 0.0 & 0.0 & 0.06 & & & 0.0 & & 53.312 & \\
\hline GSST & 0.0 & 0.0 & 0.0 & 0.0 & 0.0 & 0.0 & 0.0 & 0.0 & .0 & 0.0 & 0.0 & 0.0 & 0.020 & $20.02-2 y-3$ & 20.0 & 20.0 & 0.0 & $\begin{array}{lll}0.0 & 0.0\end{array}$ & 0.0 & 0.0 & 0.0 & 0.0 & 0.0 & 0.0 & 0.0 & 0.0 & $\begin{array}{lllll}0.0 & 0.0 & 0.0 & 0.0 & 0 .\end{array}$ & $0.0 \quad 0.0$ & $\begin{array}{ll}0 & 0.0\end{array}$ & $\begin{array}{ll}0 & 0.0\end{array}$ & 0.0 & 0.0 & 0.0 & 0.0 & 0.0 & 0.0 & 0.0 & 0.00 & 0.0 & 0.0 & 0.00 & $\begin{array}{ll}0.0 & 1\end{array}$ & 1.3 \\
\hline & 0.0 & 0.0 & 66.7 & 33.3 & 0.0 & 0 & 0.0 & 57.1 & 1.0 & & 14.3 & 0.0 & $\begin{array}{ll}.0 & 57\end{array}$ & 57.15 & 57.1 & 57.1 & 57.11 & 14.32 & & 0.0 & 14.3 & 0.0 & 0.0 & 0.0 & 0.0 & 0.0 & 0.0 & 0.014 .3 & 0.0 & 0.0 & 0.0 & 0.0 & 0.0 & 42.9 & 28.6 & 0.0 & 14.3 & & & & 0.0 & $\begin{array}{lll}0.0 & 14\end{array}$ & 14.6 \\
\hline YY & 60.0 & 60.02 & 20.01 & 100.0 & 0.0 & 0.0 & 30.0 & 10.0 & 40.0 & 20.04 & 40.08 & $80.01 \mathrm{C}$ & 100.010 & $10.0 \mathrm{I}$ & 10.0 & 10.0 & 40.05 & 50.01 & $\begin{array}{lll}0 & 0.01\end{array}$ & 100.04 & 40.03 & 30.0 & 10.0 & 30.0 & 50.0 & 30.0 & & 060.0 & .0100 .0 & 0.030 .0 & 070 & & 20.0 & 90.0 & 100.0 & 070 & & & & & & 90.051 & 51.7 \\
\hline CPC & 0.0 & 0.0 & 0.0 & 0.0 & 0.0 & 0.0 & 0.0 & 0.0 & 0.0 & 0.0 & 0.0 & 0.0 & $\begin{array}{ll}0.0 & 0 \\
-1\end{array}$ & 0.0 & 0.0 & 0.0 & 0.0 & 0.0 & 60.02 & 20.0 & 0.0 & 0.0 & 0.0 & 0.0 & 0.0 & 0.0 & 0.0 & 0.060 .6 & .00 .0 & $\begin{array}{l}0 \\
0.0\end{array}$ & 0.0 & 0.0 & 0.0 & 0.0 & 0.0 & 0.0 & 0.0 & 0.00 & 0.0 & 0.0 & 0.00 & $\begin{array}{ll}0.0 & 4 \\
Y\end{array}$ & 4.3 \\
\hline & 0.0 & & & & & & & & & & & & & & & & & & & & & & & & & 0 & & $0.0 \quad 0.0$ & 0.0 & $\begin{array}{ll}0 & 0.0\end{array}$ & 0 & 0. & 0.0 & & & 10 & 0.0 & 0.00 & 0.0 & .0 & 0.00 & 0.0 & \\
\hline & 0.0 & 0.0 & 0.0 & 0. & 0.0 & & 0.0 & 0.0 & 0.0 & 0 & 0.0 & 0.0 & $\begin{array}{ll}0.0 & 0 \\
0\end{array}$ & 0.0 & 0.0 & 0.0 & 0.0 & $\begin{array}{lll}0.0 & 0.0\end{array}$ & 0.0 & 0.0 & 0.0 & 0.0 & 0.0 & 0.0 & 0.0 & 0.0 & $\begin{array}{llllll}0.0 & 0.0 & 0.0 & 0.0 & 0 .\end{array}$ & $0.0 \quad 0.0$ & 0.0 & $\begin{array}{ll}0 & 0.0\end{array}$ & 0.0 & 0.0 & 0.0 & 0.0 & 0.0 & 0.0 & 0.0 & 0.00 & 0.0 & 0.0 & 0.00 & $0.0 \quad 0$ & 0.0 \\
\hline & 0.0 & 0.0 & 0. & & & & & & & & & & & & & & & & & 0. & & & & & & 0.0 & & $\begin{array}{ll}0.0 & 0.0 \\
-\end{array}$ & .0 & 0.0 & 0.0 & 0. & 0.0 & & 1.0 & 0.0 & 0.0 & & & & & 0.0 & 1.5 \\
\hline & 0.0 & 0.0 & 0. & 0 & 0.0 & & 0 & 0. & 0 & & 0 & 0.0 & 2.90 & 0.0 & 0.0 & 0.0 & 57.1 & 0.00 .0 & 0.0 & 0.0 & & 0.0 & 0 & 0.0 & .0 & 0.0 & $\begin{array}{cccccc}0.0 & 0.0 & 0.0 & 0.0 & 0 .\end{array}$ & $\begin{array}{ll}0.0 & 0.0 \\
-\end{array}$ & $\begin{array}{ll}0 & 0.0\end{array}$ & $\begin{array}{l}0 \\
0.0\end{array}$ & 0.0 & 0.0 & 0.0 & .0 & 0.0 & 0.0 & 0.0 & $0.0 \quad 0$ & 0.0 & 0.0 & 0.00 & 0.02 & \\
\hline & 0.0 & 0.0 & 0. & 0 & & & & 0 & & & 0.0 & 0. & & ( & & 0. & & & 0.0 & & 0. & 0 & 0 & 0 & 0 & 0 & 0 & 0.0 & 0.0 & 0 & & & & & 50.0 & & & & & & & 50.05 & 5. \\
\hline & 0.0 & 0.0 & & & & & & 0 & & $0^{0}$ & 0 & 0 & & 0 & & 0 & & 00.0 & 0.0 & 0. & & 0 & 0 & & & 0.0 & $\begin{array}{cccc}0.0 & 0.0 & 0.0 & 0 .\end{array}$ & $0.0 \quad 0.0$ & 0.0 & 0.0 & & & 0.0 & & 10 & 0.0 & 0.0 & $\begin{array}{ll}0.0 & 0\end{array}$ & 0.0 & 0. & 0 & 0.0 & 0.7 \\
\hline & 0.0 & & 0.0 & & & & 0.0 & 0 & & & & 0.0 & & & & 0. & & & & & & & & 0 & .0 & 0.0 & $\begin{array}{lll}10 & 0.0\end{array}$ & 0.0 & 0.0 & 0.0 & 0.0 & & & 0 & 0.0 & 0.0 & 0.0 & $\begin{array}{lll}0.0 & 0\end{array}$ & 0.0 & 0.0 & 0.0 & 0.0 & 2.1 \\
\hline & 7. & & & & & & 12.7 & & & & & & & & & & & & & 12.7 & & & & & & & & & & & & & & & & & & & & & & & \\
\hline
\end{tabular}

lithostratigraphic boundaries than across geochronologic boundaries in this basin. This phenomenon can be explained by the comparatively longer time-spans of geochronologic units (ranging between 1 and 15.5 million years) and lithostratigraphic formation units (ranging between 2 and 18 million years) compared with lithostratigraphic members (ranging between less than a million year and few million years). In other words, distinct geochemical signatures exist at the levels of depositional units deposited within shorter timespans than longer time-spans. This inference is supported by the sea level controlled nature of deposition in this basin and the prevalence of more or less constant sea levels $(\sim 50 \mathrm{~m})$ on a basin scale. Thus, the geochemical characteristics of the deposits of this basin are uniform on a basin scale, which vary highly at depositional units of relatively shorter time-spans.
Concerning the individual populations, the Dalmiapuram Formation shows better separation (average discrimination of $75.8 \%$ ), with almost all the elements. The Niniyur Formation deposited during Danian showed the least separation. Between different elements, silica had produced better separation of formations, perhaps because of episodic sea level changes that alternately controlled influx of detrital sediments or starvation of the depocentre. Sulphur content provided least separation of the formations. Discrimination of the lithostratigraphic members (Table 2(c)) produces very poor distinction except $100 \%$ discrimination of Odiyam member by $\mathrm{Na}, \mathrm{S}, \mathrm{As}, \mathrm{Mg} / \mathrm{Ca}, \mathrm{Fe} / \mathrm{Ca}, \mathrm{Ba} / \mathrm{Ca}, \mathrm{Na} / \mathrm{Ca}$ and $\mathrm{Zn} / \mathrm{Ca}$ and Kattupiringiyam member by $\mathrm{Mg}$, may be influenced by their distinct lithological and facies characteristics. The average discrimination $\%$ is also very low $(10.7 \%)$. 


\subsection{Discrimination of depositional units}

\subsubsection{Discrimination of chronological units}

Discrimination of chronological units with all the variables had produced $86.6 \%$ of separation (Table 3 ). Distinctness ranges between $70.8 \%$ and $100 \%$. Two chronostratigraphic units, namely Barremian and Santonian in which major tectonic movements and fluvial and fluviomarine deposition took place (Ramkumar et al, 2004), show higher discrimination percentages meaning they are highly different from other units. Although, the Kallamedu Formation which is also a fluvial deposit, is different from the Barremian and Santonian deposits in terms of its morphologically mature, but texturally immature recycled sands while the fluvial deposits of the Barremian and Santonian ages include lithoclasts

Table 3 Discrimination \% of depositional units with all the samples

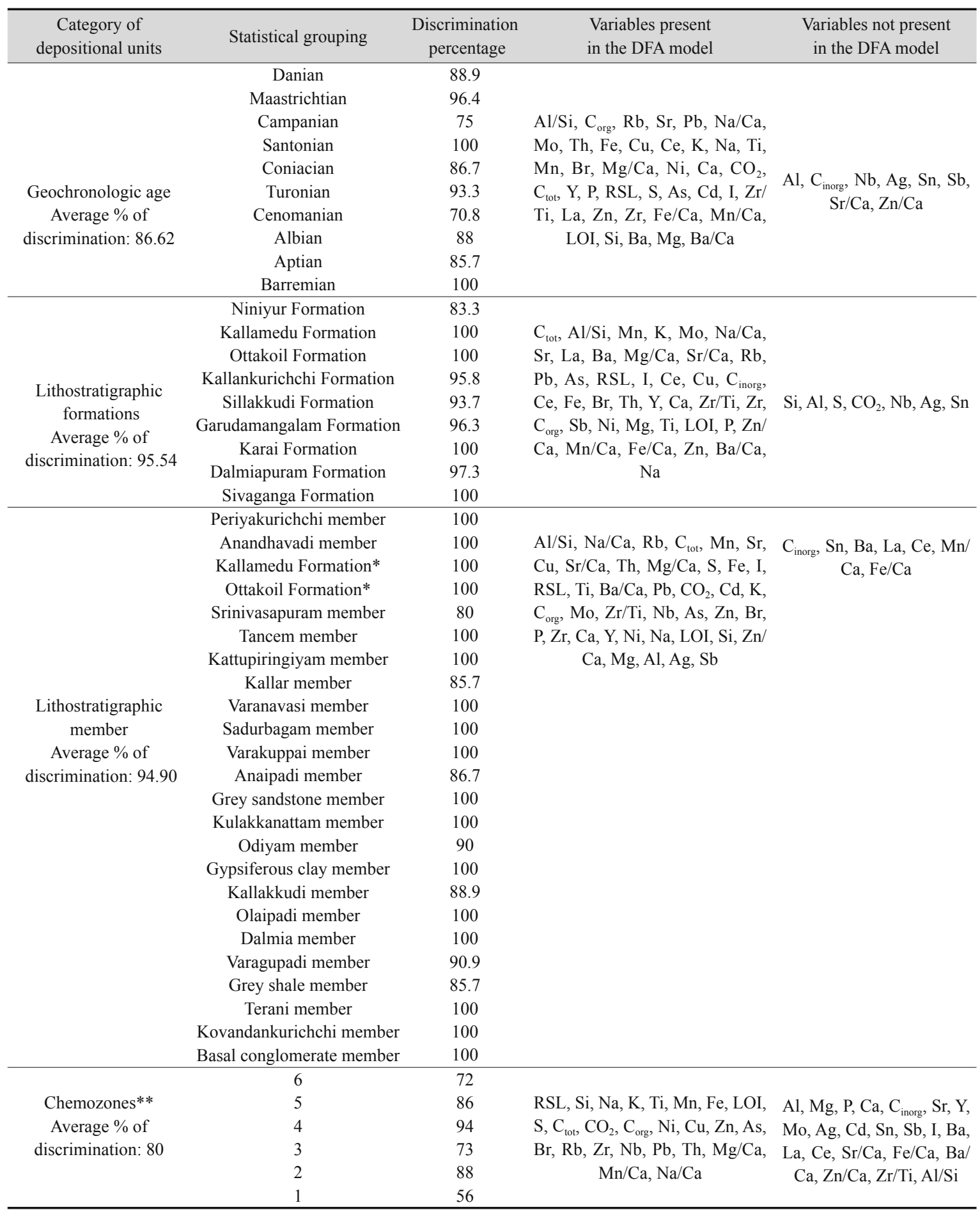

*These two formations do not have members and hence the samples of those formations were treated as members in order to test their distinctness from other members.

** Discrimination of chemozones with 70 samples had produced $100 \%$ discrimination for all the zones. 
deposited under very high energy conditions. This difference had resulted in distinction of the Barremian and Santonian deposits from all other units including that of the Kallamedu Formation, espousing the sensitivity of geochemical composition to genetic factors. In addition, the capacity of DFA to recognize this sensitivity is also demonstrated by these distinctions.

The percentages of discrimination are derived from the level of difference of constituents of each populations, measured by DFA as distances between populations, namely Squared Mahalanobis Distances (SMD). It was observed that the distances of Barremian and Santonian are relatively higher than all other populations. Cenomanian shows least discrimination percentage, may be due to the fact that it comprises a variety of rocks namely, siliciclastics, carbonates, silty and sandy clay, etc. As samples from diverse depositional settings show a variety of textural, mineralogical and geochemical compositions, they resemble other populations too, making the Cenomanian unit not distinguishable.

It is interesting to note that all the variables except $\mathrm{Al}$, $\mathrm{C}_{\text {Inorg }}, \mathrm{Nb}, \mathrm{Ag}, \mathrm{Sn}, \mathrm{Sb}, \mathrm{Sr} / \mathrm{Ca}$ and $\mathrm{Zn} / \mathrm{Ca}$ show distinct variation between the chronological units (Table 3). Presence of all the parameters except these few emphasize that distinction of chronological units as espoused by DFA is a cumulative effect of all those elements in the list, not dependent on a single or few elemental concentrations. It could be interpreted such that as the sea level changes have brought in differential combinations of depositional sequences through time, their signatures are embedded on almost all the elemental concentrations too, albeit with differential impact owing to varied sensitivities of elements, nature of detrital components, depositional conditions and climate, etc. The presence of $\mathrm{Al} / \mathrm{Si}$ ratio in the list of discriminant variables indicates that the relative influx of mature sediments (quartz) and immature sediments (quartz and feldspar or quartz, feldspar and clay) would have played a role in differentiating these populations. It also emphasizes the interpretation made earlier that the initial depositional history was dominated by immature sediments and through passage of time, sediment to be transported to the depocentre might have become scarce, forcing the depositional agents to recycle older deposits. Occurrences of $\mathrm{C}_{\mathrm{Org}}$, $\mathrm{Sr}$ and RSL (relative sea level), in the list of variables that discriminate all the formations emphasize the dominant control exercised by sea level variations over depositional processes.

\subsubsection{Discrimination of lithostratigraphic units}

Discrimination of formations produces an average of $95.5 \%$ distinctness (Table 3). Minimum of $83.3 \%$ and maximum of $100 \%$ distinctness and many populations with $100 \%$ discrimination are recorded, meaning discrimination percentages of individual formations are higher than that of chronological units. Among the variables that discriminate these populations, presence of $\mathrm{C}_{\mathrm{Tot}}$ and $\mathrm{Al} / \mathrm{Si}$ indicates the control on carbonate deposition by detrital influx and variation of detrital materials in terms of different mineral associations like, quartz or quartz + feldspar or quartz + feldspar + clay with reference to different formations. This observation is supported by the list of variables that have not shown any distinctness wherein $\mathrm{Si}, \mathrm{Al}, \mathrm{S}, \mathrm{CO}_{2}, \mathrm{Nb}, \mathrm{Ag}$ and
$\mathrm{Sn}$ are present. It means that the individual concentrations of Si and Al do not show any distinctness; rather, it is their relative abundance that allows the depositional unit to be distinguished or not. RSL also shows significant distinctness among different formations, confirming the role of sea level variations through the depositional history of the basin. SMD of discrimination of formation has shown that all the formations except the Dalmiapuram Formation have more or less equal distances/distinctness from each other. Performing DFA of lithostratigraphic members has produced distinctness of $94.9 \%$ (Table 3). Discrimination of individual members ranges from $80 \%$ to $100 \%$. Most of the members (17 out of 24) show a distinctness of $100 \%$. The presence of RSL in the discriminating variables list also confirms the observation of the sea level controlled depositional pattern in this basin. This might have brought in differential depositional conditions, as a result of which varied lithofacies associations were developed. All these in turn have differential geochemical compositions to the level of $100 \%$ distinct.

Andrew et al (1996) observed that recognizable chemostratigraphic units appeared to be lithologically distinctive in the Surat Basin, Australia. Based on integrated sequence and chemostratigraphic studies on sedimentary sequences of the Siberian craton, Pelechaty et al (1996) found correspondence between isotopic and geochemical shifts at the scale of individual depositional units. Variations in gross lithology/lithofacies association and trace element chemistry of sediments induced by changes in climate and sea level oscillations have been recorded by Pearce and Jarvis (1992). They also observed that geochemically distinct depositional units agree with distinct mineralogical and grain size variations. Pearce et al (1999) reported coeval boundaries of lithostratigraphic and chemostratigraphic units. Wignall and Newton (2001) recorded coincidence of lithostratigraphic boundary with sequence boundary. Sarg (1988) stated that four major variables namely, tectonic subsidence, eustatic sea level change, volume of sediments and climate control stratal patterns and lithofacies distributions. He has also demonstrated how the sea level changes affect and overwhelm the influences of other variables. Observations of these authors, together with the distinctness of bulk chemistry of the depositional units that were deposited in the Cauvery Basin under the influence of global scale climatic and sea level fluctuations, clearly portray the preservation of depositional features. As observed by many authors cited herein, these results also indicate the presence of distinct changes in the chemical signatures of depositional units at lithostratigraphic boundaries.

While comparing the discrimination of chronological units and lithostratigraphic units, it became evident that the lithostratigraphic units show more or less similar distinction (95.5\% for formations and $94.9 \%$ for members) while the chronological units show lower discrimination (86.6\%). When these observations are coupled with the influence of sea level changes over depositional regimes, they lead to the interpretation that prevalent sea level changes inscribed their imprints on depositional sequences (sensu stricto Vail et al, 1977) vis-à-vis lithostratigraphic units more than on chronological units. This interpretation also supports the need 
to elucidate bulk geochemical signatures of depositional units and treating those units as chemozones when geochemical profiles carry subtle and complex signals. Now, the question remains over reasons for the absence of $100 \%$ discrimination for all the units. If the signatures recognized through statistical discrimination are to be used for recognizing the affiliations of unknown samples, the signatures should prove themselves to be $100 \%$ accurate in the first place. Only then could any further classification be made with confidence. One potential source for the absence of $100 \%$ discrimination could be that, some of the samples have not been analyzed for few geochemical constituents (namely, $\mathrm{Si}, \mathrm{Al}, \mathrm{Na}, \mathrm{Mg}, \mathrm{P}$ and LOI). To test the validity of this assumption, DFA was performed with 70 samples that were analyzed for a more complete set of major and trace elemental concentrations. The results show $100 \%$ average success with all the populations having $100 \%$ distinction. SMD values for chronological units, formations and members are presented in the Tables 4(a), 4(b) and 4(c), respectively. While comparing these tables, it can be seen that the distances show a general increase from chronological units to members. It means that the geochemical signatures of these depositional units are strongly discriminative at member level and weak at chronological unit level. Considering the geochemical signatures of individual units, the shift in values of geochemical parameters is drastic at boundaries of depositional units that have least chronological span (lithostratigraphic members) and the changes diminish when the time span is increased. This may be the reason for invisible/subtle/complex signals expressed by geochemical profiles. Such an observation raises concern over potential flaws while delineating the chemozones based essentially on manual examination of profiles and trend lines. Present observations, together with the interpretations of linear and polynomial trend lines made by Ramkumar et al (in press, 2010) justify the search for alternative method, as practiced in this study, for delineation of chemozones, particularly when long range strata are studied.

Table 4(a) SMD of discrimination of chronological units with 70 samples

\begin{tabular}{l|cccccccccc}
\hline & DN & MA & CA & SA & CO & TU & CE & AL & AP & BA \\
\hline DN & 0.00 & & & & & & & & & \\
MA & 69.06 & 0.00 & & & & & & & & \\
CA & 138.07 & 62.08 & 0.00 & & & & & & & \\
SA & 197.76 & 208.04 & 176.97 & 0.00 & & & & & & \\
CO & 196.44 & 117.54 & 88.05 & 245.22 & 0.00 & & & & & \\
TU & 140.74 & 169.84 & 188.69 & 351.50 & 275.24 & 0.00 & & & & \\
CE & 83.22 & 57.10 & 93.01 & 255.17 & 170.30 & 96.06 & 0.00 & & & \\
AL & 92.67 & 65.20 & 102.24 & 247.51 & 200.80 & 101.04 & 30.82 & 0.00 & & \\
AP & 118.24 & 97.92 & 119.15 & 230.29 & 204.21 & 183.52 & 79.54 & 70.78 & 0.00 & \\
BA & 532.78 & 478.30 & 457.43 & 673.44 & 558.49 & 481.99 & 408.30 & 459.74 & 545.02 & 0.00 \\
\hline
\end{tabular}

Table 4(b) SMD of discrimination of formations with 70 samples

\begin{tabular}{|c|c|c|c|c|c|c|c|c|}
\hline & NIN & KMU & OTK & KLN & SIL & GRD & KRI & DLM SVG \\
\hline NIN & 0.00 & & & & & & & \\
\hline KMU & 1011.33 & 0.00 & & & & & & \\
\hline OTK & 546.26 & 623.52 & 0.00 & & & & & \\
\hline KLN & 45.83 & 1148.02 & 553.51 & 0.00 & & & & \\
\hline SIL & 149.33 & 1264.64 & 599.62 & 118.64 & 0.00 & & & \\
\hline GRD & 125.96 & 1155.72 & 620.38 & 119.01 & 102.85 & 0.00 & & \\
\hline KRI & 114.54 & 1169.45 & 578.98 & 83.21 & 77.15 & 45.49 & 0.00 & \\
\hline DLM & 39.64 & 1093.05 & 535.32 & 33.66 & 94.48 & 71.52 & 49.83 & 0.00 \\
\hline SVG & 110.16 & 1376.47 & 782.26 & 86.86 & 118.42 & 184.29 & 125.92 & 93.130 .00 \\
\hline
\end{tabular}

Table 4(c) SMD of discrimination of members with 70 samples

\begin{tabular}{|c|c|c|c|c|c|c|c|c|c|c|c|c|c|c|c|c|c|c|c|c|c|c|c|c|}
\hline & PERY & ANDY & KMDU & OTKL & SRNI & TNCM & KAPM & KALR & VARN & SADR & VRKP & ANAP & GSST & KLKM & ODYM & GYPC & KLKK & OLPD & DALM & VGPD & GREY & TRNY & KVDN & BASL \\
\hline PERY & 0 & & & & & & & & & & & & & & & & & & & & & & & \\
\hline ANDY & 30473 & 0 & & & & & & & & & & & & & & & & & & & & & & \\
\hline KMDU & 7386 & 44606 & 0 & & & & & & & & & & & & & & & & & & & & & \\
\hline OTKL & 4220 & 42056 & 3415 & 0 & & & & & & & & & & & & & & & & & & & & \\
\hline SRNI & 1544 & 20785 & 12555 & 8994 & 0 & & & & & & & & & & & & & & & & & & & \\
\hline TNCM & 3414 & 14884 & 13130 & 9333 & 1395 & 0 & & & & & & & & & & & & & & & & & & \\
\hline KAPM & 10819 & 6569 & 25864 & 21597 & 4844 & 3077 & 0 & & & & & & & & & & & & & & & & & \\
\hline KALR & 2583 & 17978 & 11333 & 8348 & 1107 & 364 & 4330 & 0 & & & & & & & & & & & & & & & & \\
\hline VARN & 2346 & 36084 & 13671 & 9796 & 2664 & 6685 & 12920 & 5440 & 0 & & & & & & & & & & & & & & & \\
\hline SADR & 4728 & 14639 & 11332 & 10084 & 3009 & 1840 & 4636 & 2272 & 8934 & 0 & & & & & & & & & & & & & & \\
\hline VRKP & 8181 & 7967 & 17461 & 15539 & 4243 & 1912 & 1803 & 2863 & 12501 & 1485 & 0 & & & & & & & & & & & & & \\
\hline ANAP & 7216 & 9753 & 18133 & 14183 & 3332 & 988 & 1426 & 1793 & 10267 & 2399 & 1055 & 0 & & & & & & & & & & & & \\
\hline GSST & 20129 & 11569 & 43411 & 36957 & 11320 & 11340 & 4369 & 13186 & 17510 & 14656 & 10564 & 8545 & 0 & & & & & & & & & & & \\
\hline KLKM & 43247 & 2580 & 62974 & 58393 & 30699 & 23682 & 11674 & 27474 & 47178 & 24954 & 16275 & 16501 & 12121 & 0 & & & & & & & & & & \\
\hline ODYM & 31580 & 9896 & 57570 & 50165 & 19982 & 18145 & 7706 & 20823 & 29002 & 22248 & 15568 & 13402 & 1785 & 7442 & 0 & & & & & & & & & \\
\hline GYPC & 4825 & 15910 & 10760 & 8031 & 3949 & 1400 & 5582 & 1669 & 10770 & 2004 & 2179 & 2285 & 18028 & 26509 & 25308 & 0 & & & & & & & & \\
\hline KLKK & 784 & 37189 & 4972 & 2144 & 4157 & 6055 & 15891 & 4874 & 4297 & 6866 & 11494 & 10728 & 28057 & 52180 & 41072 & 6033 & 0 & & & & & & & \\
\hline OLPD & 514 & 27437 & 7596 & 5165 & 1361 & 3190 & 9304 & 2489 & 2992 & 3454 & 6585 & 6278 & 18740 & 40046 & 29785 & 4121 & 1399 & 0 & & & & & & \\
\hline DALM & 2908 & 15393 & 14129 & 10229 & 613 & 527 & 2683 & 829 & 5252 & 1877 & 2241 & 1455 & 9384 & 24158 & 16722 & 2527 & 5834 & 2375 & 0 & & & & & \\
\hline VGPD & 707 & 23459 & 8158 & 4928 & 1066 & 1426 & 7249 & 1011 & 4067 & 2662 & 4738 & 4099 & 17169 & 35147 & 26883 & 2095 & 1832 & 724 & 1376 & 0 & & & & \\
\hline GREY & 2571 & 17526 & 11133 & 6732 & 1727 & 750 & 4803 & 1204 & 6766 & 1705 & 2872 & 2066 & 14033 & 27530 & 22099 & 1363 & 4362 & 2135 & 837 & 941 & 0 & & & \\
\hline TRNY & 66043 & 24601 & 99069 & 91843 & 48760 & 44698 & 28108 & 47785 & 60324 & 50996 & 39953 & 36556 & 16774 & 14955 & 9138 & 54556 & 79562 & 63674 & 44338 & 59215 & 52571 & 0 & & \\
\hline KVDN & 391 & 27907 & 8789 & 5605 & 1057 & 3064 & 9296 & 2366 & 2315 & 4330 & 6972 & 6553 & 18045 & 40500 & 28781 & 4592 & 1519 & 594 & 2381 & 653 & 2568 & 62543 & 0 & \\
\hline BASL & 43290 & 142675 & 34488 & 34786 & 58902 & 68136 & 96205 & 62563 & 44129 & 69524 & 85051 & 82620 & 115369 & 170675 & 142436 & 67916 & 35557 & 47269 & 67743 & 52198 & 63487 & 201855 & 46905 & 0 \\
\hline
\end{tabular}




\subsubsection{Discrimination of major chemozones}

Collation of the results and the review presented above of the role of sea level fluctuations over lithofacies (Table 5) necessitated examination of the stratigraphic variation of geochemistry in resonance with depositional history. The sea level curve of this basin (Fig. 2) shows the presence of six third order cycles. These cycles are separated by type I sequence boundaries (recognized through shift of shoreline crossing shelf break evidenced by contact relationship between strata, subaerial exposure, widespread subaerial erosion and advancement of fluvial channels over former offshore regions, etc., Ramkumar et al, 2004), confirming that sea level controlled the depositional pattern in this basin. This interpretation is further supported by the presence of global sea level peaks in this basin (Raju et al, 1993) namely, at $104 \mathrm{MY}$ (Early-Late Albian), 93.7 MY $( \pm 0.9$; Middle to Late Cenomanian), 92.5 MY ( \pm 1; Early to Middle Turonian),
86.9 MY ( \pm 0.5 ; Early to Late Coniacian), $85.5 \mathrm{MY}( \pm 1$; Early to Late Santonian), $73 \mathrm{MY}$ ( \pm 1 ; Late Campanian), 69.4 MY (Early to Late Maastrichtian) and $63 \mathrm{MY}( \pm 0.5$; Early to Middle Danian).

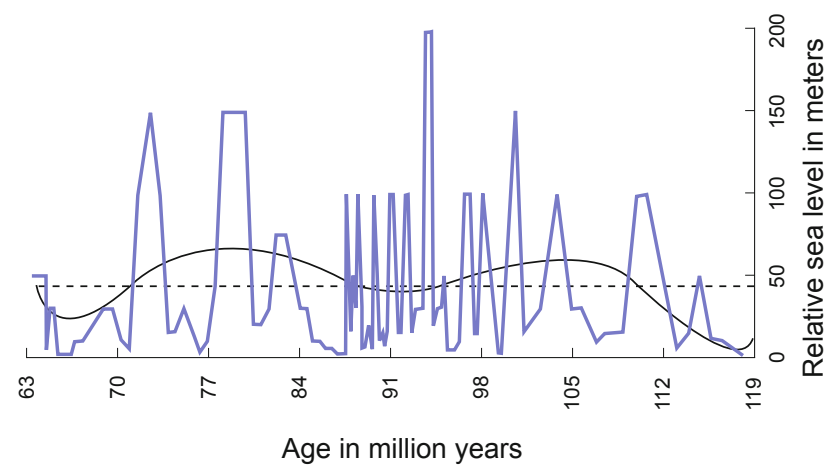

Fig. 2 Inferred sea level cycles

Table 5 Depositional units, breaks and events in the Cauvery Basin

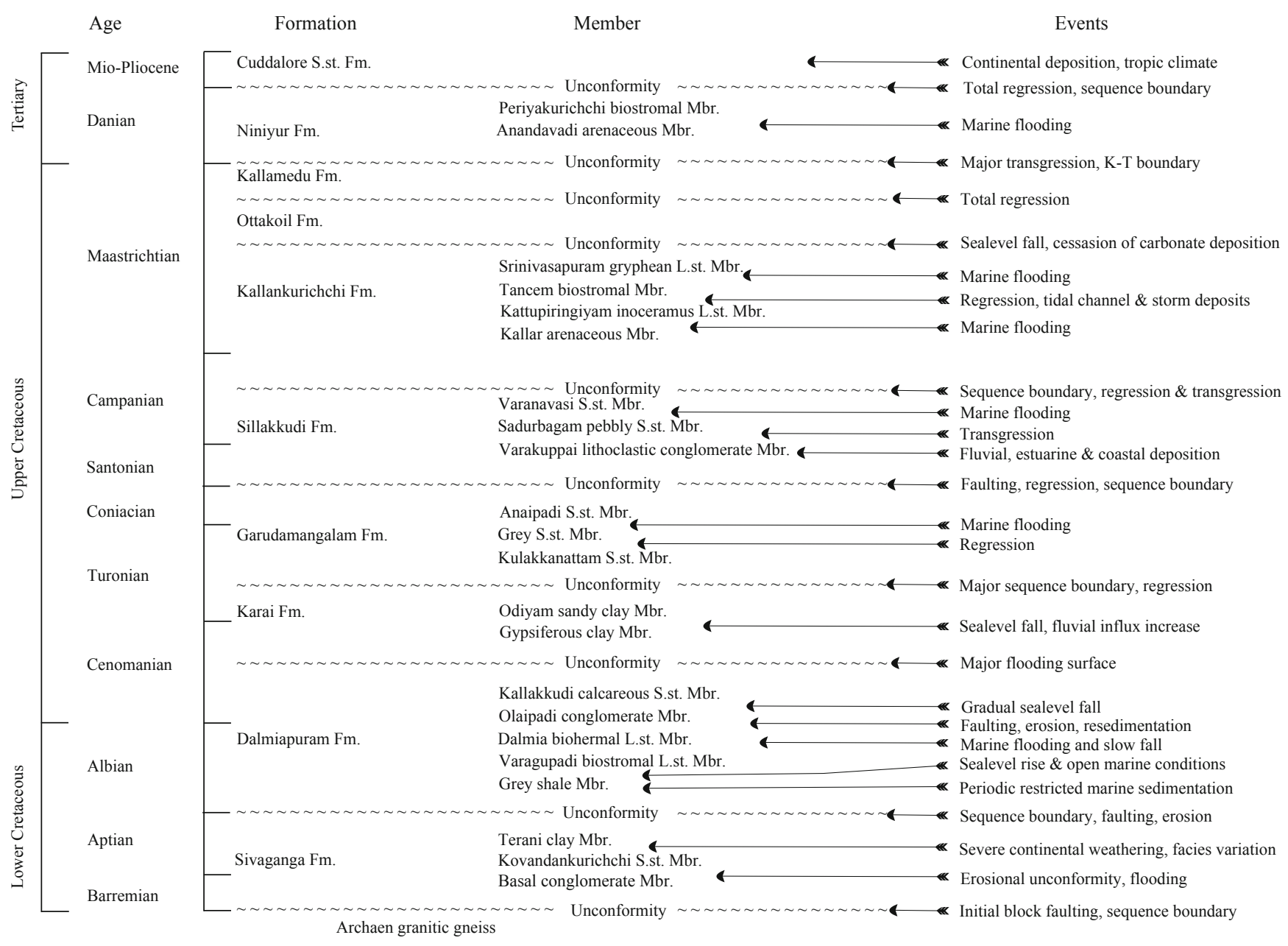

Examination of the profiles of $\mathrm{Si}$ and $\mathrm{Ca}$ (Fig. 3) in the light of these observations shows that six zones of enrichment/depletion can be recognized. Many of the trace elements show affinity with either of these two elemental concentrations (Ramkumar et al, 2006) and hence, delineation of major chemozones based on $\mathrm{Si}$ and $\mathrm{Ca}$ is thus justified. It is also observed that within these major zones there exist many high frequency cycles of enrichment and depletion. To verify the existences of six cycles vis-à-vis chemozones, the assignment of samples to these provisional chemozones has been attempted. The results confirm the presence of six major chemozones with a fully discriminative shift of bulk composition at their boundaries that was influenced by $3^{\text {rd }}$ order sea level cycles. The list of variables that serve as 
discriminants includes all the elements and measured ratios except $\mathrm{LOI}, \mathrm{CO}_{2}, \mathrm{C}_{\text {Inorg }}, \mathrm{Ni}, \mathrm{Th}, \mathrm{Al} / \mathrm{Si}$ which means, the chemozones recognized with the help of trends of $\mathrm{Ca}$ and $\mathrm{Si}$ and third order sea level cycles are indeed followed by all other elemental concentrations except these six constituents. Presence of LOI, $\mathrm{CO}_{2}, \mathrm{C}_{\text {Inorg }}$ in the list of constituents that do not serve as discriminants could indicate uniform carbonate sedimentation with reference to major chemozones. Their insignificance in discrimination of chemozones but their significant difference between chronological and lithostratigraphic units suggests that carbonate sedimentation has had a differential response to high frequency cycles and or differential temporal resolution. Presence of elements, $\mathrm{Ni}$ and Th that get adsorbed onto clay mineral surfaces in the list of constituents that do not serve as discriminants may indicate that they might have undergone remobilization. However, their presence in discrimination of other depositional units signifies their distinct change at differential temporal resolution. Similarly, presence of $\mathrm{Al} / \mathrm{Si}$ could indicate nonsignificance of relative abundance/influx of quartz, feldspar or clay at the temporal scales of third order cycles. Collectively, these inferences support utilization of a large number of elemental compositions for chemostratigraphic studies. These inferences also suggest chemostratigraphic analysis at various temporal scales, as the geochemistry varies as a function of different depositional controls and processes.
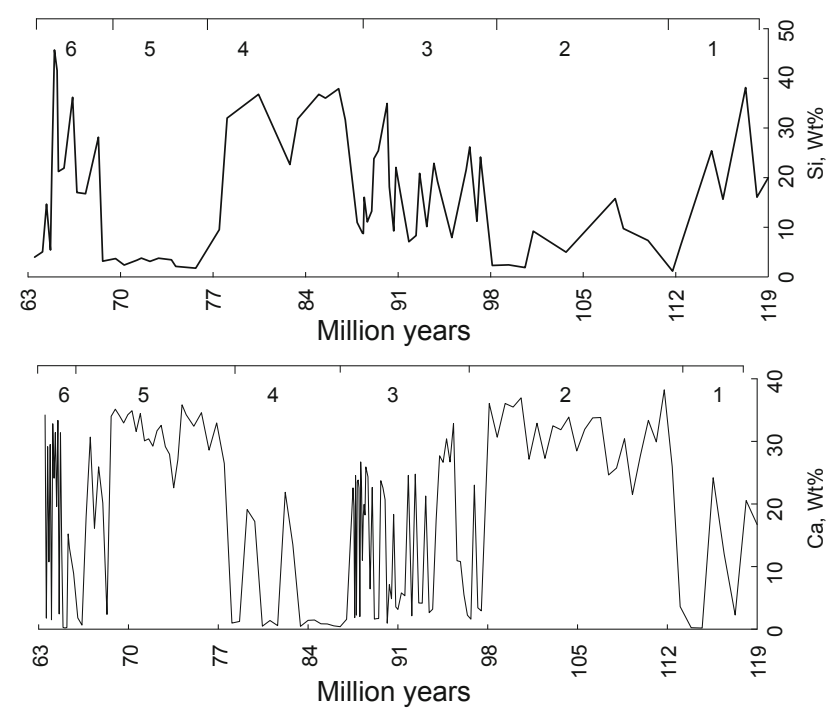

Fig. 3 Zones of Silica and Calcium enrichment and depletion

\section{Implications on spatio-temporal scales of correlation}

Results of discrimination of depositional units have shown the existence of statistically tested $100 \%$ geochemical disparity between them with reference to almost all the chemical elements studied, which in turn are not observable through manual examination and delineation in geochemical profiles and trends. DFA has shown the presence of subtle, but statistically robust shifts in geochemical compositions as a result of genetic differences of those units. Occurrences of distinct geochemical shifts at lithostratigraphic member levels, presence of many high frequency cycles in geochemical profiles and sea level curve, prevalent control of sea level over depositional pattern call for recognition of chemozones within lithostratigraphic members. Recognizing such short-term cycles/high-resolution chemozones would be difficult owing to limitations of the dataset. However, this limitation does not preclude the assumption on the existence of such cycles which can be discriminated as depositional units in terms of geochronological unit (maximum of 18 million year cycle) and member $(<1$ million year cycle), which have proved to have $100 \%$ distinct geochemical signatures. As such, individual members could be considered as distinct chemozones with the available data.

Perfect correspondence of the six major chemozones with six $3^{\text {rd }}$ order sea level cycles and lithostratigraphic members with $4^{\text {th }}$ order sea level cycles (Ramkumar et al, 2006; in press, 2010) calls for further analysis. The introduction of the concept of global sea level changes and their control over stratal patterns and facies distribution and the fact that such changes are recorded in sedimentary geochemistry give rise to the possibility of correlating stratigraphic records with their counterparts located elsewhere. The relative sea level cycles, first published by Vail et al (1977) and revised by Haq et al (1987) espoused that sedimentary sequences are produced principally under the influence of sea level cycles that vary between few tens of millions of years ( $1^{\text {st }}$ order cycle) to few million years $\left(3^{\text {rd }}\right.$ order cycle). Successive studies have shown that distinct sedimentary sequences could be traced to sea level cycles up to infra seventh order (Carter et al, 1991). Vail et al (1977) stated that the sea level chart published by them is incomplete and cycles of varying order could be added as the studies on sedimentary sequences progress, so that a more complete chart could be produced. The aim behind this statement is to incorporate sea level cycles at the Milankovitch scale, to which the response of the sedimentation system is proved beyond reasonable doubt (Carter et al, 1991). Hays et al (1976) convincingly demonstrated that climatic records were dominated by frequencies characteristics of variations in the Earth's tilt, precession and eccentricity relative to the Sun. In the years since, numerous studies have upheld the validity of the Milankovitch climatic cycles in terms of 100 , $41,23 \mathrm{Ka}$ orbital periods that influence or control variations in global ice volume, thermohaline circulation, continental aridity and run off, sea surface temperature, deep ocean carbonate preservation and atmospheric $\mathrm{CO}_{2}$ and methane concentrations (Raymo et al, 1997). Cyclic sedimentation has been documented in numerous sedimentary basins and there are many lines of evidences that relate those cycles to shortterm (Milankovitch band) glacio-eustatic pulses (Grammer et al, 1996). Global chemostratigraphic signals such as those carried by organic matter (Calver, 2000) oxygen and strontium isotopes (Veizer et al, 1999) and their relationships with sea level changes are well known. The global carbon cycle varies on a million year time scale affecting the isotopic and chemical composition of the global carbon (Wallmann, 2001). The glacial intervals coincide with shifts in $\delta^{18} \mathrm{O}$ and $\delta^{13} \mathrm{C}$. For the carbon isotope record, the rate of burial of $\mathrm{C}_{\mathrm{Org}}$ and thereby changes in atmospheric $\mathrm{CO}_{2}$ and for the oxygen isotopic records, temperature and ice volume effects 
on the seawater reservoirs and thereby sea level changes may be linked (Kampschulte et al, 2001). Spectral analysis of $\delta^{18} \mathrm{O}$ and $\delta^{13} \mathrm{C}$ shows that their significant variances are concentrated at 100, 43, 23 and $19 \mathrm{Ka}$ spans (Oppo et al, 1990). While examining $\delta^{18} \mathrm{O}$ of Phanerozoic seawater, Veizer et al (1997) observed the presence of high frequency cycles within the first order cycle. Strauss (1997) recorded $4^{\text {th }}$ order cycles of sulphur isotope that stack up to form $3^{\text {rd }}$ order cycle fluctuations that in turn accommodated within $2^{\text {nd }}$ order cycles. Goldhammer et al (1991) showed that the sequences of the Paradox Basin exhibited a distinct cyclicity characterized by a hierarchical stacking pattern such that, $5^{\text {th }}$ order shallowing upward cycles group into $4^{\text {th }}$ order cycles, which in turn stack vertically into part of a $3^{\text {rd }}$ order cycle.

The observations and views of many authors reviewed above give rise to two inferences. The first is that the stratigraphic records are influenced by climatic and thus sea level changes, periodicities of which vary from few thousand years to tens of million years. The second is that the ensuing sediments do record such changes in terms of varying nature of geochemical compositions. In this context, interpretation of six chemozones corresponding to $3^{\text {rd }}$ order sea level cycles in the Cauvery Basin, together with the results of Ramkumar et al (2004; 2006; in press, 2010) that the sea level curve of this basin expresses the presence of fourth order sea level cycles coinciding with lithostratigraphic members, that stack up to third order cycles which in turn form part of second order cycles. This stacking-up nature indicates that the distinct signatures may have been influenced by global climatic and sea level fluctuations. It also raises the possibility of existence of many higher order cycles within lithostratigraphic members, which in turn has to be verified through additional sampling coverage. Occurrences of poor discrimination of depositional units with reference to individual elements and $100 \%$ disparity when all the elements reaffirm the view that bulk composition of the rocks could serve the purpose for chemostratigraphic studies and geochemical characterization of depositional units in terms of lithostratigraphic and chronological divisions.

\section{Conclusions}

1) Existence of statistically $100 \%$ distinct shift/change of bulk geochemical composition of the strata at the scales of geological age, lithostratigraphic formations and members in the Cauvery Basin, south India has been documented. These disparities could not be observed through conventional visual observation of geochemical profiles of the same data. Statistical analyses and interpretations helped recognize six major chemozones.

2) Sedimentation history of the basin was predominantly controlled by sea level changes, resulting in distinct lithofacies and corresponding bulk geochemical compositions. As all the global sea level peaks are recognized in this basin and the sea level cycles recognized were of glacioeustasy type (Carter et al, 1991), the cycles of geochemical compositions might have been influenced by global signals. The geochemical zones range from less than a million to 18 million years. These are major geochemical cycles, within which multiple higher order cycles are embedded, similar to the stacking patterns of depositional sequences.

3) It is found that examination of groups of elements for high-resolution chemostratigraphic signatures gives more clues than examination of individual elemental concentrations. In addition, examination of the data through statistical discrimination would be accurate and reliable.

4) In order to recognize chemozones in long ranging strata, sampling according to detailed lithostratigraphic and chronostratigraphic units, elucidation of controls on elemental chemistry and association of elements are necessary before dividing the strata based on geochemical trends.

5) Results of this study have implications on scales of stratigraphic correlation; i.e., once a distinct geochemical signature of a particular depositional unit is recognized and tested to have statistical uniqueness, the signature could be utilized for recognizing similar unit elsewhere at an appropriate scale depending on the extent of the signal being local or regional, etc. This postulate is based on the uniqueness of geochemical signatures observed in the data of the present study. Its veracity can only be tested when compatible datasets from other regions or basins are available.

\section{Acknowledgements}

We have benefited much from the constructive suggestions and modifications offered by the anonymous reviewers that have helped us presenting the data and interpretations in a lucid way. MR acknowledges the financial assistance of Alexander von Humboldt Foundation, Germany. Council of Scientific and Industrial Research, New Delhi, India provided financial support during field surveys. Currently research on chemostratigraphy of the Cauvery Basin is supported by Department of Science and Technology, New Delhi. Special thanks are due to the institute scientific personnel namely, Dr. Utz Kramar for TXRF analytical facilities, Dr. Karotke and Mrs. Oetzel for XRD analyses, Mr. Zrinkjack for carbon and sulphur analyses and Dr. Ott for computing facilities. Shri. T. Sreekumar, Geologist, OFI, Mumbai is thanked for assistance during the field survey. Permission to collect samples was accorded by the mines managers and geologists of Messers. Alagappa Cements, Chettiyar Mines, Dalmia Cements Pvt. Ltd., Fixit Mines, Nataraj Ceramics Ltd., Parveen Mines and Minerals Ltd., Ramco Cements, Rasi Cements, TANCEM Mines, TAMIN Mines, Tan-India Mines, and Vijay Cements.

\section{References}

Andrew A S, Whitford D J, Hamilton P J, et al. Application of chemostratigraphy to petroleum exploration and field appraisal: An example from the Surat Basin. Proceedings of Seminar on Asia Pacific Oil and Gas. 1996. 421-429

Calver C R. Isotope stratigraphy of the Ediacarian (Neoproterozoic III) of the Adelaide rift complex, Australia, and the overprint of water column stratification. Precambrian Research. 2000. 100(1): 121-150

Carter R M, Abbott S T, Fulthorpe C S, et al. Application of global sealevel and sequence stratigraphic models in Southern Hemisphere Neogene strata from New Zealand. Inter. Assoc. Sediment. Spl. Publ. 1991. 12: 41-65

Das N. Chemostratigraphy of sedimentary sequences: A review of the state of the art. Journal of Geological Society of India. 1997. 49: 


\section{1-628}

Ferrell R E, Hart G F, Swamy S, et al. X-Ray mineralogical discrimination of depositional environments of the Krishna Delta, Peninsular India. Journal of Sedimentary Research. 1998. 68(1): 148154

Goldhammer R K, Oswald E J and Dunn P A. Hierarchy of stratigraphic forcing: Example from middle Pennsyl-vanian shelf carbonates of the Paradox Basin. In: Franseen E K, Watney W L, Kendall C St C, et al, eds., Sedimentary Modeling. Kansas Geological Survey Bulletin. 1991. 233: 361-413

Govindan A, Ananthanarayanan S and Vijayalakshmi K G. Cretaceous petroleum system in Cauvery Basin, India. In: Govindan A, eds., Cretaceous stratigraphy: An update. Memoirs of Geological Society of India. 2000. 46: 365-382

Grammer G M, Eberli G P, Van Buchem F S P, et al. Application of highresolution sequence stratigraphy to evaluate lateral variability in outcrop and subsurface-Desert Creek and Ismay intervals, Paradox Basin. In: Longman M W and Sonnenfeld M D, eds., Paleozoic Systems of the Rocky Mountain Region. Society of Economic Palaeontologists and Mineralogists. 1996. 235-266

Haq B U, Hardenbol J and Vail P R. Chronology of fluctuating sea levels since the Triassic. Science. 1987. 235(4793): 1156-1167

Hays J D, Imbrie J and Shackleton N J. Variations in the earth's orbit: Pacemaker of the ice ages. Science. 1976. 194(4270): 1121-1132

Kampschulte A, Bruckschen P and Strauss H. The sulphur isotope composition of trace sulphates in Carboniferous brachiopods: implications for coeval seawater, correlation with other geochemical cycles and isotope stratigraphy. Chemical Geology. 2001. 175(1): 149-173

Loizeau J L and Stanley D J. Petrological-statistical approach to interpret recent and subrecent lagoon sub-facies, Idku, Nile delta of Egypt. Marine Geology. 1993. 111: 55-81

Nayak B J, Rajev S M and Sahoo R K. R-mode factor analysis and its implications on the geochemistry of Nishikhal manganese deposit, Raiguda district, Orissa. Journal of Geological Society of India. 1997. 49: 133-144

Oppo D W, Fairbanks R G, Gordon A L, et al. Late Pleistocene southern ocean $\delta^{13} \mathrm{C}$ variability. Paleoceanography. 1990. 5(1): 43-54

Pearce T H and Jarvis I. Applications of geochemical data to modeling sediment dispersal patterns in distal turbidites: late Quaternary of the Madeira abyssal plain. Journal of Sedimentary Research. 1992. 62(6): 1112-1129

Pearce T J, Besly B M, Wray D S, et al. Chemostratigraphy: a method to improve interwell correlation in barren sequences - a case study using onshore Duckmantian/Stephanian sequences (West Midlands, U.K.). Sedimentary Geology. 1999. 124(1): 197-220

Pelechaty S M, Grotzinger J P, Kashirtsev V A, et al. Chemostratigraphic and sequence stratigraphic constraints on Vendian-Cambrian basin dynamics, Northeast Siberian Craton. Journal of Geology. 1996. 104: 543-563

Prabhakar K N and Zutshi P L. Evolution of southern part of Indian east coast basins. Journal of Geological Society of India. 1993. 41. 215230

Raju D S N, Ravindran C N and Kalyansundar R. Cretaceous cycles of sea level changes in Cauvery Basin, India - A first revision. Oil and Natural Gas Corporation Bulletin. 1993. 30: 101-113

Ramkumar M. Role of chemostratigraphic technique in reservoir characterisation and global stratigraphic correlation. Indian Journal of Geochemistry. 1999. 14: 33-45

Ramkumar M. Sedimentary microenvironments of modern Godavari delta: characterization and statistical discrimination-Towards computer assisted environment recognition scheme. Journal of
Geological Society of India. 2001. 57: 49-63

Ramkumar M and Guha A K. Multivariate statistical verification of petrographic and standard microfacies types and lithostratigraphy of Tertiary carbonates of western Kutchch, Gujarat, India: Implications on global stratigraphic correlation and hydrocarbon exploration. Indian Journal of Petroleum Geology. 2000. 9: 52-74

Ramkumar M and Sathish G. Integrated sequence and chemostratigraphic modelling: A sure-fire technique for stratigraphic correlation, petroleum exploration and reservoir characterization. In: Rajendran S, Srinivasamoorty K and Aravindan S, eds., Mineral Exploration: Recent Strategies. New India Publishers. 2006. 21-40

Ramkumar M, Berner Z and Stüben D. Multivariate statistical discrimination of selected carbonate petrographic classifications: Implications on applicability of classification systems and predictability of petrographic types. International Journal of Geochemistry. 2002. 62(2): 145-159

Ramkumar M, Stüben D and Berner Z. Lithostratigraphy, depositional history and sea level changes of the Cauvery Basin, southern India. Annals of Geology of Balkan Peninsula. 2004. 65: 1-27

Ramkumar M, Stüben D and Berner Z. Elemental interrelationships and depositional controls of Barremian-Danian strata of the Cauvery Basin, south India: Implications on scales of chemostratigraphic modelling. Indian Journal of Geochemistry. 2006. 21: 341-367

Ramkumar M, Stüben D and Berner Z. Barremian-Danian chemostratigraphic sequences of the Barremian-Danian strata of the Cauvery Basin, south India: Implications on stratigraphic correlation. Gondwana Research. 2010 (in press)

Raymo M E, Oppo D W and Curry W. The mid-Pleistocene climate transition: A deep sea carbon isotopic perspective. Paleoceanography. 1997. 12(4): 546-559

Roser B P and Korsch R J. Provenance signatures of sandstonemudstone suits determined using discriminant function analysis of major element data. Chemical Geology. 1988. 67(1-2): 119-139

Sarg J F. Carbonate sequence stratigraphy. In: Sea level changes: An integrated approach. Society of Economic Palaeontologists and Mineralogists Special Publication. 1988. 42: 155-181

Strauss H. The isotopic composition of sedimentary sulphur through time. Palaeogeography, Palaeoclimatology, Palaeoecology. 1997. 132: $97-118$

Stüben D, Kramar U, Berner Z, et al. Trace elements, stable isotopes and clay mineralogy of the Elles II K-T boundary section in Tunisia: indications for sea level fluctuations and primary productivity. Palaeogeography, Palaeoclimatology, Palaeoecology. 2002. 178(3): 321-345

Vail P R, Mitchum R M and Thompson S. Seismic stratigraphy and global changes of sea level. Part 4: Global cycles of relative changes of sea level. In: Payton C E, eds., Seismic stratigraphy_Applications to hydrocarbon exploration. Memoirs of American Association of Petroleum Geologists. 1977. 26: 83-97

Veizer J, Ala D, Azmy K, et al. ${ }^{87} \mathrm{Sr} r{ }^{86} \mathrm{Sr}, \delta^{13} \mathrm{C}$ and $\delta^{18} \mathrm{O}$ evolution of Phanerozoic seawater. Chemical Geology. 1999. 161(1): 59-88

Veizer J, Bruckschen P, Pawellek F, et al. Oxygen isotope evolution of Phanerozoic seawater. Palaeogeography, Palaeoclimatology, Palaeoecology. 1997. 132(1): 159-172

Wallmann K. Controls on the Cretaceous and Cenozoic evolution of seawater composition, atmospheric $\mathrm{CO}_{2}$ and climate. Geochimica et Cosmochimica Acta. 2001. 65(18): 3005-3025

Wignall P B and Newton R. Black shales on the basin margin: a model based on examples from the upper Jurassic of the Boulonnais, northern France. Sedimentary Geology. 2001. 144(3): 335-356

(Edited by Hao Jie) 